\title{
Nuevos cementos eco-eficientes elaborados con la fracción fina de hormigón reciclado para futuras aplicaciones ingenieriles
}

\author{
M. Monasterio ${ }^{1 *}$, M. Frías ${ }^{1}$, R. García ${ }^{2}$, R. Vigil de la Villa ${ }^{2}$, S. Martínez ${ }^{3}$, L. Fernández ${ }^{4}$, E. \\ Lahoz1, I. Vegas ${ }^{5}$, J. Moreno ${ }^{5}$ \\ *Autor de Contacto: manuel.monasterio@ietcc.csic.es \\ ${ }^{1}$ Eduardo Torroja Institute for Construction Science (IETcc-CSIC), 28033 Madrid, Spain \\ ${ }^{2}$ 2Dept. of Geology and Geochemistry, 'Geomateriales', CSIC-UAM Associated Research Unit, Autonomous \\ University of Madrid, Madrid, Spain \\ ${ }^{3}$ Institute for the Structure of Matter (IEM-CSIC), 28006 Madrid, Spain \\ ${ }^{4}$ Department of Civil and Environmental Engineering, Barcelona TECH, 08034 Barcelona, Spain \\ ${ }^{5}$ Tecnalia, Basque Research and Technology Alliance (BRTA), Astondo Bidea, Edificio 700, Parque Tecnológico \\ de Bizkaia, 48160, Derio, Spain
}

RESUMEN

Los residuos de la construcción son un gran problema medioambiental. Europa ha priorizado su reciclaje para nuevas construcciones ingenieriles. Sin embargo, la fracción fina de los residuos de hormigón, no presenta aplicaciones y son almacenados en las plantas de tratamiento. Este trabajo investiga el comportamiento de pastas de cemento elaboradas con un 7\% de la fracción fina de hormigón de diferente naturaleza. Los resultados muestran que estos residuos presentan poca actividad puzolánica y sus propiedades físicas y mecánicas son muy similares a la pasta de cemento. A pesar de ello, los cementos mezcla cumplirían con la normativa para cementos comunes. Esto abre una vía para reutilizar estos residuos en nuevas tecnologías de construcción como puede ser impresión 3D.

Palabras clave: Fracciones finas de hormigón reciclado; caracterización; propiedades de las pastas de cemento mezcla; nuevas aplicaciones ingenieriles.

\section{RESUMO}


Resíduos de construção são um grande problema ambiental. A Europa priorizou sua reciclagem para novas construções de engenharia. No entanto, a fração fina dos resíduos de concreto não tem aplicação e é armazenada em estações de tratamento. Este trabalho investiga o comportamento de pastas de cimento feitas com $7 \%$ da fração fina de concreto de diferentes naturezas. Os resultados mostram que esses resíduos apresentam pouca atividade pozolânica e suas propriedades físicas e mecânicas são muito semelhantes às da pasta de cimento. Apesar disso, os cimentos mistos estariam em conformidade com os regulamentos para cimentos comuns. Isso abre uma maneira de reaproveitar esses resíduos em novas tecnologias de construção, como a impressão 3D.

Palavras-chave: Frações finas de concreto reciclado; caracterização; propriedades de pastas de cimento; novas aplicações de engenharia.

\begin{abstract}
The construction wastes are a big environmental problem. Europe has prioritized its recycling for new engineering constructions. However, the fine fraction of concrete waste has no applications and is stored in treatment plants. This work researches the behavior of cement pastes made with $7 \%$ of the concrete fine fraction from different nature. The results show that these residues have little pozzolanic activity and their physical and mechanical properties are very similar to cement paste. Despite this, the mixed cements would fulfill the regulations for common cements. This opens a way to reuse this waste in new construction technologies such as $3 \mathrm{D}$ printing.
\end{abstract}

Keywords: Recycled concrete fine fraction; characterization; mixed paste cement properties; new engineering applications

\title{
1. INTRODUCCIÓN
}

La industria de la construcción es uno de los mayores motores del desarrollo socio-económico de un país. Sin embargo, está directamente relacionada con impactos negativos tanto en el medioambiente, en el cambio climático o en el consumo de energía[1]. Además de ello, una gran cantidad de residuos de construcción y demolición (RCDs), son generados al final de la vida útil de las estructuras ingenieriles, edificación, etc., acumulándose, en la mayoría de los casos en vertederos e incluso en vertidos ilegales, provocando importantes problemas técnicos, económicos, medioambientales y de salud[2]. En el 2016, solo en Europa se generó alrededor de 374 Mt/año de RCDs[3], de los cuales, únicamente se recicló el 50\%, la mayor parte en aplicaciones de bajo valor añadido, como relleno en carreteras[4]. Partiendo de esta base, hay un gran espacio de mejora en cuanto al aprovechamiento de estos RCDs como materiales de alto valor añadido, campo que Europa ha identificado como una prioridad[5]. Parte de esta prioridad es el enorme potencial que presentan estos RCDs para el cumplimiento de los objetivos de desarrollo sostenible(ODS) y de la economía circular, recuperando estos residuos como materias primas secundarias para el propio sector de la construcción[6,7].

Con este objetivo, el aprovechamiento de estos residuos, por su heterogeneidad, pasa por las plantas de tratamientos de RCDs para la selección de los diferentes materiales presentes: hormigón reciclado, cerámico, mixto, papel, plásticos, madera, hierro, etc,[8-12]. Uno de estos residuos RCDs que están centrando especial interés en la comunidad científica son los residuos de hormigón reciclado, por estar recogidos en diferentes instrucciones y recomendaciones como árido grueso 
reciclado en la fabricación de nuevos hormigones ecológicos[13]. Sin embargo, la preparación de estas fracciones granulométricas de árido grueso reciclado genera una facción fina $(<5 \mathrm{~mm})$, que se acumula en las propias instalaciones de las plantas de tratamiento, bajo la influencia de las condiciones atmosféricas, con propiedades adversas (alta absorción de agua, impurezas, etc) que hacen difícil su reutilización en la industria de la construcción[14].

Los estudios previos han puesto de manifiesto la viabilidad de utilizarse como nuevos materiales cementantes suplementarios[15,16] con los consiguientes impactos en la producción de matrices de cemento con menor contenido en clinker e influir en el cumplimiento de la neutralidad de $\mathrm{CO}_{2}$ para el 2050[17].

Por este motivo, en el presente trabajo se profundiza en esta línea de investigación, analizando dos tipos de residuos de hormigón reciclado (calcáreo y silíceo), como sustitución parcial del cemento OPC. Para ello, se analiza las propiedades químicas, físicas, mineralógicas y puzolánicas de estos residuos industriales, así como su comportamiento en las nuevos cementos eco-eficientes y sostenibles con las políticas medioambientales.

\section{MATERIALES Y MÉTODOS}

\subsection{Materiales}

Dos tipos de residuos de fracción fina de hormigón $(<5 \mathrm{~mm})$ procedentes de RCDs fueron analizados en la presente investigación: Un residuo de hormigón de naturaleza silíceo (HsT), procede de hormigones fabricados con árido silíceo, el cual fue suministrado por la planta de tratamiento TECREC, Ubicada en la Comunidad de Madrid (España) localizada en el centro de España (Tecrec, Madrid, España) y un segundo residuo de hormigón de naturaleza calcáreo (HcG) procedentes de hormigones elaborados con árido calcáreo, suministrado por la planta de tratamiento GUTRAM, ubicada en la comunidad Vasca (España).

El residuo de fracción fina se obtiene después del proceso de trituración y tamizado del hormigón reciclado en las plantas de tratamiento para la obtención de fracciones granulométricas gruesas para distintos usos industriales (árido reciclado para hormigones, árido para carreteras, etc.).

Estos residuos finos presentan un inconvenientes técnico y medioambiental ya que son acumulados en las propias plantas de tratamiento sin ninguna utilidad industrial en la actualidad. Sin embargo, por su naturaleza pueden ser viables para la fabricación de eco-cementos. Estos residuos finos, una vez secados en una estufa a $105^{\circ} \mathrm{C}$ durante 1 hora, se sometieron a un proceso de trituración y tamizado hasta conseguir tamaños de partícula inferiores a $63 \mu \mathrm{m}$, para obtener finuras similares a los del cemento OPC comercial. En la Figura 1 se pueden observar los dos residuos, una vez finalizado este proceso previo a su empleo como sustituto del cemento.

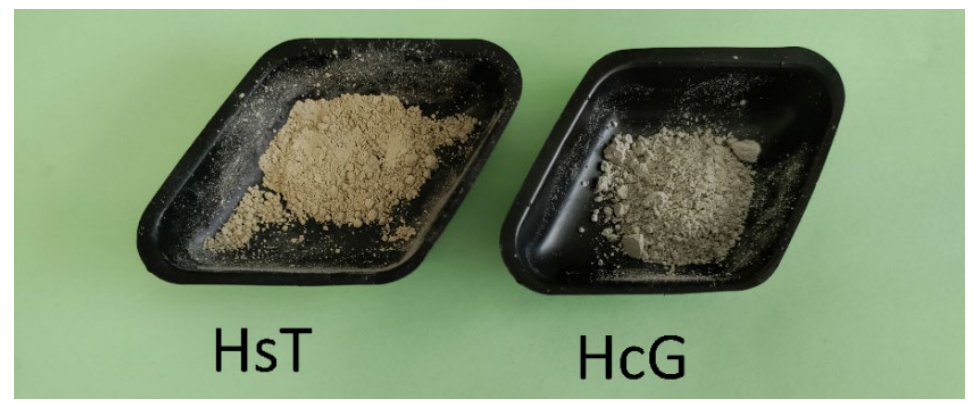

Figura 1. Imagen de los dos residuos empleados en estre trabajo, HsT y HcG de origen silíceo y calcáreo, respectivamente, después del proceso de triturado y tamizado. 
El cemento portland comercial utilizado (OPC) es del tipo CEM I $52.5 \mathrm{R}$, suministrado por Cementos Lemona, S.A. (Bilbao, España). Su composición mineralógica obtenida por Rietveld mostró una composición mineralógica de $52 \%$ de $\mathrm{C}_{3} \mathrm{~S}, 20 \%$ de $\mathrm{C}_{2} \mathrm{~S}, 9 \%$ de $\mathrm{C}_{3} \mathrm{~A}, 6 \%$ de $\mathrm{C}_{4} \mathrm{AF}, 4 \%$ de calcita y finalmente, un $9 \%$ de fase amorfa.

\subsection{Métodos}

\section{Preparación de las matrices de cemento}

Las pastas y los morteros fueron elaborados por la sustitución parcial del cemento OPC por un 7\% de cada una de cada una de las fracciones finas de hormigón, porcentaje óptimo según trabajos previos $[15,18]$.

Las pastas de cemento mezcla fueron preparadas de acuerdo a la norma europea EN 196-1[19], empleando $500 \mathrm{~g}$ de mezcla de cemento con una relación agua/mezcla de 0.5 . Después, las probetas fueron fabricadas en moldes prismáticos de 1 x 1 × $6 \mathrm{~cm}$ y compactadas de acuerdo a la normativa vigente [20]. Posteriormente, las probetas de pasta de cemento fueron desmoldadas después de 24 horas y curadas en agua durante 2, 28 y 90 días.

\section{Actividad puzolánica}

La evaluación de la actividad puzolánica fue llevada a cabo mediante un método químico acelerado, consistente en añadir $1 \mathrm{~g}$ de residuo a $75 \mathrm{~mL}$ de solución saturada de cal $\left(\mathrm{Ca}(\mathrm{OH})_{2}\right)$ y mantenerlo a $40{ }^{\circ} \mathrm{C}$ hasta finalización del ensayo [21]. Al final de cada edad, la disolución fue filtrada y la concentración de calcio en la solución fue determinada por volumetría con EDTA. El porcentaje de cal fijada por el residuo era calculado por diferencia con la disolución control realizada en las mismas condiciones de ensayo.

\section{Demandas de agua, expansión y tiempo de fraguado}

Las medidas de ensayo de consistencia normal, estabilidad de volumen y tiempos de fraguado se realizaron tal y como se describe en la norma europea EN-196-3[22].

\section{Comportamiento mecánico}

La resistencia a la flexión de las pastas fue medida en un NETZSCH (Netzsch, Selb, Alemania). Seis muestras prismáticas de 1 x 1x $6 \mathrm{~cm}$ fueron ensayadas para cada tipo de cemento y edad de curado. Para la resistencia a la compresión se empleó un IBERTEST AUTOTEST 200/20-SW (Ibertest, Madrid, España), de acuerdo a la normativa EN 196-1 2018[23].

\subsection{Técnicas Instrumentales}

La composición química de los materiales de partida fue realizada por fluorescencia de rayos X, en un Philips PW-1404 spectrometer (Phillips, Madrid, España), ajustado con un tubo de rayos X Sc-Mo.

La distribución de tamaño de particular fue analizada por la técnica de difracción de rayos laser[24] con un equipo Malvern Mastersizer 3000 analyser (Malvern Panalytical, Madrid, España) equipado con fuentes de luz roja y azul (He-Ne y LED) en modo de dispersión seca. El rango de medida fue entre 0.01 y $3500 \mu \mathrm{m}$.

El análisis mineralógico fue llevado a cabo por difracción de rayos X en un PAN Analytical X'Pert Pro X-ray diffractometer (Malvern Panalytical, Davis, CA, USA), ajustado con un ánodo de $\mathrm{Cu}$, operando a $40 \mathrm{~mA}, 45 \mathrm{kV}$, usando una ranura de divergencia de 0.50 y una ranura de recepción de $0.5 \mathrm{~mm}$. Las muestras se escanearon en un rango de $2 \theta$ entre 50 y 600 , con saltos de ángulo de 0.0167 (20) a 150 ms/paso. El Rutilo fue usado como patrón interno. La cuantificación por Rietveld fue evaluada usando Match v.3 y FullProf suite (Crystal Impact, Bonn, Alemania) y las fases mineralógicas se identificaron usando la Crystallography Open Database (COD). 
La microporosidad de las pastas endurecidas fue determinada por intrusión de mercurio en un Micromeritics Autopore IV porosimeter (Micromeritics, Norcross, GA, USA). Este dispositivo opera a presiones que alcanzan los 33,000 psi (227.5 MPa), llegando a medir diámetros de poro entre 0.006 y $175 \mu \mathrm{m}$.

\section{RESULTADOS Y DISCUSIÓN}

\subsection{Caracterización química de las muestras de partida}

En la Tabla 1 se recoge las composiciones químicas obtenidas por FRX, expresadas en óxidos. Se observa, como era de esperar, que cuantitativamente las fracciones finas HsT y HcG presenten composiciones químicas diferentes, pero que cualitativamente ambas son similares entre si y similares a la del cemento portland comercial (OPC), ya que los óxidos mayoritarios son $\mathrm{SiO}_{2}$, $\mathrm{Al}_{2} \mathrm{O}_{3}$ y CaO. El residuo HsT presenta una gran cantidad de $\mathrm{SiO}_{2}$ y bajo contenido en $\mathrm{CaO}$, mientras que el $\mathrm{HcG}$ está formado mayoritariamente por $\mathrm{CaO}$ y bajo contenido en $\mathrm{SiO}_{2}$. Las composiciones químicas obtenidas están de acuerdo con trabajos previos realizados por otros autores, con materiales de partida de origen similar[25]. Además, la perdida al fuego muestra unos valores muy altos, probablemente por la descomposición de carbonatos y fases hidratadas en los hormigones originales.

Tabla 1. Composicion quimica de los materiales de partida

\begin{tabular}{|c|c|c|c|}
\hline Oxido & OPC & HsT & HcG \\
\hline $\mathrm{SiO}_{2}$ & 41.22 & 49.97 & 9.34 \\
\hline $\mathrm{Al}_{2} \mathrm{O}_{3}$ & 2.89 & 8.98 & 2.88 \\
\hline $\mathrm{CaO}$ & 69.81 & 18.65 & 50.32 \\
\hline $\mathrm{Fe}_{2} \mathrm{O}_{3}$ & 3.70 & 2.30 & 1.20 \\
\hline $\mathrm{MgO}$ & 0.93 & 1.37 & 1.12 \\
\hline $\mathrm{SO}_{3}$ & 3.36 & 2.53 & 0.85 \\
\hline $\mathrm{Na}_{2} \mathrm{O}$ & 0.33 & 0.80 & 0.18 \\
\hline $\mathrm{K}_{2} \mathrm{O}$ & 0.76 & 3.35 & 0.47 \\
\hline $\mathrm{P}_{2} \mathrm{O}_{5}$ & 0.14 & 0.11 & 0.03 \\
\hline $\mathrm{TiO}_{2}$ & 0.20 & 0.28 & 0.14 \\
\hline $\mathrm{MnO}$ & 0.10 & 0.04 & 0.09 \\
\hline $\mathrm{PPC}$ & 3.22 & 11.50 & 33.20 \\
\hline
\end{tabular}

En cuanto a la composición mineralógica obtenida por DRX-Rietveld, como se puede observar en la Tabla 2, los dos residuos de hormigón presentan las mismas fases mineralógicas, pero en distintas proporciones cristalinas. En la muestra HsT, el cuarzo es la fase cristalina dominante, como de era de esperar por su origen, mientras que en $\mathrm{HcG}$, la fase dominante es la calcita, igualmente debido a su origen.

Tabla 2. Composición mineralógica por DRX- Rietveld de los residuos HsT y HcG. $\mathrm{R}_{\mathrm{b}}$ representa el factor R de Bragg y $\mathrm{X}^{2}$ la finura del ajuste.

\begin{tabular}{|c|c|c|c|c|c|c|c|}
\hline Material & $\begin{array}{c}\text { Mica } \\
(\mathbf{\%})\end{array}$ & $\begin{array}{c}\text { Cuarzo } \\
(\mathbf{\%})\end{array}$ & $\begin{array}{c}\text { Feldespato } \\
(\mathbf{\%})\end{array}$ & $\begin{array}{c}\text { Calcita } \\
(\mathbf{\%})\end{array}$ & $\begin{array}{c}\text { Fase amorfa } \\
(\mathbf{\%})\end{array}$ & $\mathbf{R}_{\mathbf{B}}$ & $\mathbf{X}^{\mathbf{2}}$ \\
\hline $\mathrm{HsT}$ & 4 & 48 & 8 & 24 & 16 & 17.6 & 7.3 \\
\hline $\mathrm{HcG}$ & 10 & 10 & 11 & 52 & 17 & 23.9 & 6.9 \\
\hline
\end{tabular}


Las curvas de densidad de distribución para los 3 materiales de partida, se muestran en la Figura 2. Los residuos de hormigón HsT y HcG, muestran una distribución bimolar con máximos entre 32 $-38 \mu \mathrm{m}$ y $6-7 \mu \mathrm{m}$, con diferentes intensidades de bandas, debido a su diferente contenido de fases mineralógicas cristalinas (calcita y cuarzo) cuya resistencia a la molienda es diferente. El OPC muestra un comportamiento más próximo a una curva molal, cuyo máximo se localiza a $20 \mu \mathrm{m}$ y un ligero hombro solapado a la banda principal, localizándose a $8 \mu \mathrm{m}$ y que correspondería a la presencia de yeso como regulador de fraguado y algo de calcita en el cemento comercial. Los valores de Dx correspondientes a los diámetros por los que pasa el 10, 50 y 90\% de las partículas analizadas en cada caso, se recogen en la Tabla 3.

Tabla 3. Valores Dx de las medidas laser de finura en los materiales de partida

\begin{tabular}{|c|c|c|c|}
\hline MICRAS & HST & HCG & OPC \\
\hline D(10) & 1.04 & 0.82 & 1.99 \\
\hline D(50) & 8.42 & 5.77 & 11.6 \\
\hline D(90) & 44.9 & 31.9 & 34.2 \\
\hline
\end{tabular}

En ella se destaca la similitud de las finuras para os tres materiales de partida, propiedad física importante para el comportamiento posterior de las matrices de cemento

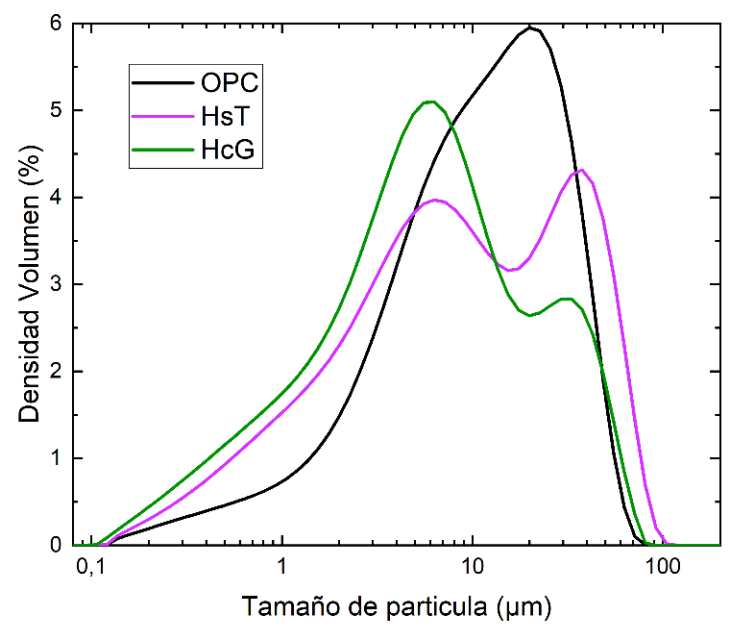

Figura 2. Distribución del tamaño de partícula para los materiales de partida obtenido por difracción laser

\subsection{Comportamiento puzolánico de las fracciones finas de hormigón reciclado}

En la Figura 3 se representa los valores de la cal fijada por los residuos HsT y HcG en el sistema puzolana. El residuo calizo HcG muestra una baja capacidad para fijar la cal del medio, debido a la presencia mayoritaria de caliza, bien conocida por su nula reactividad puzolánica y bajo contenido en sílice y alúmina. Durante los primeros 90 días de reacción, el HcG solo consume entre el 10-20\% de la cal disponible y aumenta ligeramente hasta el 32\% a los 360 días.

Por otro lado, el residuo de hormigón silíceo, con altos contenidos en óxidos ácidos (sílice y alúmina) alcanza a fijar un 35\% de cal en las primeras 24 horas de reacción, elevando este valor hasta $73 \%$ a los 180 días. A pesar de ser un valor razonablemente medio-bajo, según el tipo de residuo, hay que tener en cuenta que no se equipara con puzolanas convencionales, como el humo 
de sílice o la ceniza volante, que pueden llegar a alcanzar valores de $80 \%$ a los 90 días [26]. La moderada reactividad del residuo $\mathrm{HsT}$ a pesar de su contenido en $\mathrm{SiO}_{2}$, es debido a que esta se encuentra en la muestra en forma de cuarzo, estado cristalino sin propiedades puzolánicas.

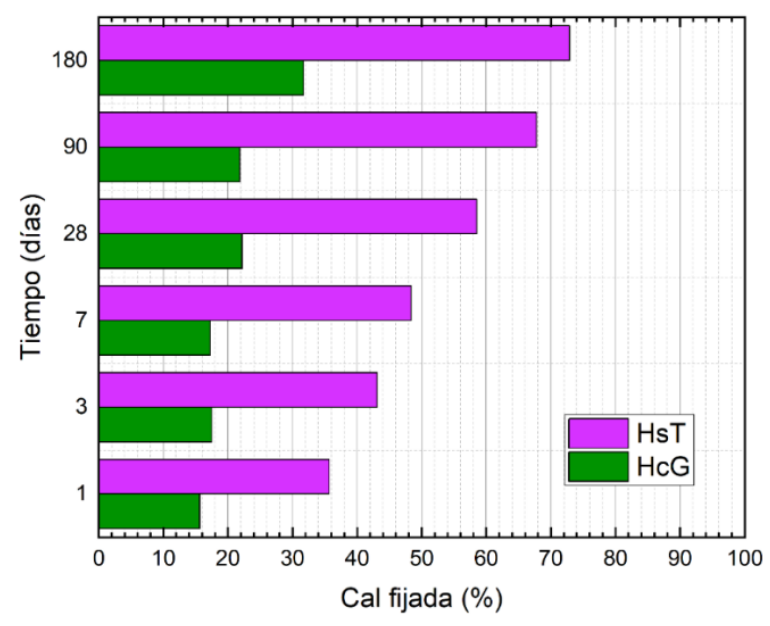

Figura 3. Actividad puzolánica de las fracciones finas de hormigón en un sistema puzolana/cal.

Un análisis cinético de la reacción puzolánica, aplicando el modelo cinético difusivo como lo describe Villar-Cociña et al. [6,7], permite la determinación de la constante de reacción K basándose en la evolución del consumo de cal con el tiempo, se obtuvieron valores de $\mathrm{K}$ de $1.18 \cdot 10^{-4}$ y $6.58 \cdot 10^{-4}$ para HcG y HsT, respectivamente. Estos valores están alejados cuando se comparan con puzolanas altamente reactivas como cenizas de hoja de bambú $\left(8.41 \cdot 10^{-1}\right)$ y cenizas de cascar de arroz $\left(1.73 \cdot 10^{-2}\right)$ [27], pero se asemejan a otros RCDs estudiados, como RCDs de naturaleza cerámica $\left(6.19 \cdot 10^{-4}\right)[28]$.

\section{3 productos de hidratación de las pastas de cemento}

Las pastas de cementos curadas a 90 días fueron analizadas por XRD para determinar los cambios mineralógicos cristalinos originados durante la reacción puzolánica en el sistema puzolana/cal. Los correspondientes difractogramas se muestran en la Figura 4. A través de ella, se identifican las principales fases cristalinas:

- La aparición de un pico muy pronunciado debido al cuarzo (Q), en la pasta 7\% HsT, localizado los picos de reflexión a 20.9, 26.6, 36.5 y 39.4 20, debido a la presencia de árido silíceo en el hormigón reciclado.

- Un incremento en la señal de la calcita (C) en la muestra con $\mathrm{HcG}$, debido a la presencia de esta fase en el árido original en el hormigón de origen, identificando claramente a 29.4 $2 \theta$.

El resto de las fases mineralógicas, procedentes de las reacciones de hidratación del cemento y puzolánica, identificadas a 90 días de reacción son: portlandita $(\mathrm{P})$, etringita (Et) o monocarboaluminato $(\mathrm{Mc})$ están presentes en todos los cementos analizados. Por otro lado, todavía se identifican la presencia de fases anhidras del propio cemento después de 90 días de hidratación como silicato tricálcico $\left(\mathrm{C}_{3} \mathrm{~S}\right)$ y silicato dicálcico $\left(\mathrm{C}_{2} \mathrm{~S}\right)$ y algo de ferrito aluminato tetracálcico $\left(\mathrm{C}_{4} \mathrm{AF}\right)[16]$. 


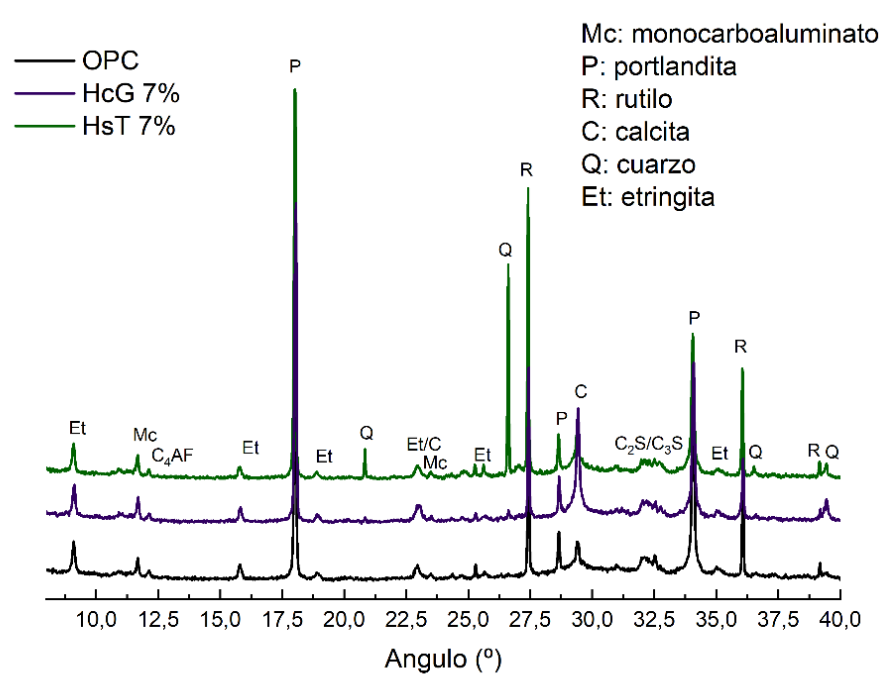

Figura 4. Difractogramas de DRX de las pastas de cemento a 90 días de hidratación. R patrón interno.

\subsection{Propiedades físicas}

Para analizar el comportamiento reológico de las pastas de cemento seleccionadas en estado fresco, los estudios se centraron en la demanda de agua para obtener una consistencia normal, fraguados y expansión de volumen, de acuerdo a la normativa vigente sobre ensayos y especificaciones físicas para los cementos comunes[29]. Los valores obtenidos en las diferentes pruebas pueden verse en la Tabla 4.

Con respecto a las demandas de agua, una ligera disminución del agua requerida se observa para las muestras con sustitución del 7\% para cada una de las fracciones finas de hormigón procedentes de los RCDs. Esta disminución es inferior al 3\% con respecto al valor de la pasta OPC de referencia, por lo que se puede considerar despreciable si se tiene en cuenta el erro del ensayo $( \pm 1 \mathrm{~g})$.

Tabla 4. Valores de los resultados del comportamiento reológico en estado fresco de las pastas de cemento con un $7 \%$ de sustitución.

\begin{tabular}{|c|c|c|c|}
\hline & OPC & HsT 7\% & HcG 7\% \\
\hline Demanda de agua $(\mathrm{g})$ & 154 & 152 & 152 \\
\hline Expansión (mm) & 0.5 & 0.5 & 0.0 \\
\hline Fraguado inicial (min) & 180 & 165 & 175 \\
\hline Fraguado final (min) & 269 & 226 & 231 \\
\hline
\end{tabular}

De forma similar, el estudio del ensayo de expansión de las diferentes pastas de cemento es prácticamente despreciable y muy similar al del OPC. Este hecho indica que la adición del 7\% de este tipo de residuo de hormigón no aporta posibles compuestos expansivos como cal y magnesia libre. Estos valores encontrados son muy pequeños comparados con el valor máximo recogido en la norma $(\leq 10 \mathrm{~mm})[29]$.

Finalmente, Los valores para los fraguados, inicial y final, son muy parecidos a la de la pasta de cemento OPC, si se tiene en cuenta el error del ensayo ( $\pm 10 \mathrm{~min})$. En cualquier caso, la norma europea solo establece limitación para el fraguado inicial mayor a 45- $60 \mathrm{~min}$ [29], por lo que cumplirían con este requerimiento físico normativo. 
Con respecto a los valores de fraguado final, se puede apreciar un efecto acelerador en este parámetro cuando se adiciona las diferentes fracciones finas de hormigón reciclado, del orden de 43 y 38 minutos para HsT 7\% y HcG 7\%, respectivamente. Esto hecho, podría estar relacionado con la presencia de calcita en las residuos [30].

\subsection{Distribución de tamaño de poro}

Los resultados de la distribución de tamaño de poro para las pastas de cemento curadas a 90 días se pueden ver en la Figura 5. Se observa como la mayor parte de los tamaños de poro en los 3 cementos están por debajo de $0.1 \mu \mathrm{m}$, aunque ligeramente desplazadas hacia microporos más grandes las pastas de cemento elaboradas con $7 \%$ de residuos de hormigón reciclado. Las pastas de cementos binarios se localizan el máximo de distribución en torno a $0.05 \mu \mathrm{m}$, mientras que la pasta OPC de referencia a $0.04 \mu \mathrm{m}$. A pesar de ello la diferencia es mínima, si se tiene en cuanta los valores de porosidad total recogidos en la Tabla 5.

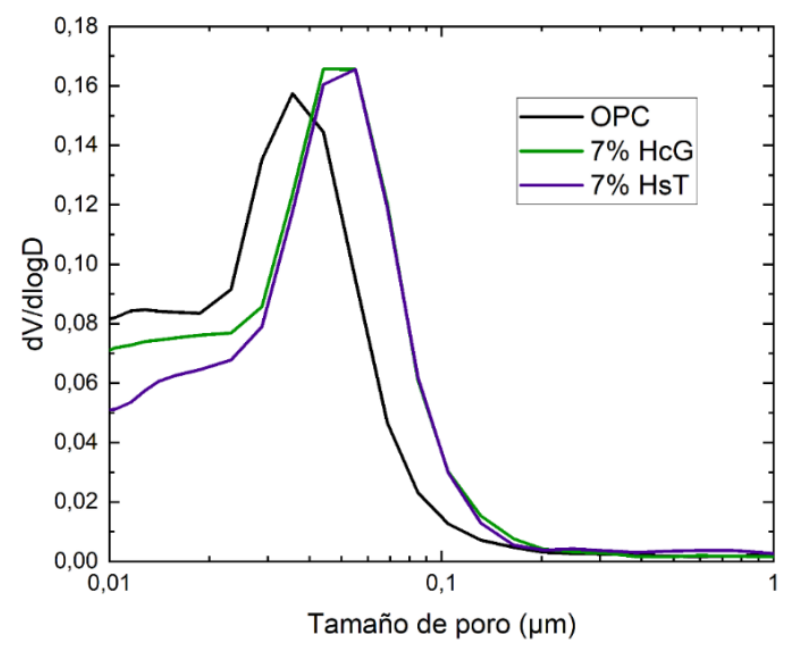

Figura 5. Distribución de tamaño de poro de las pastas de cemento estudiadas después de 90 días de curado.

Tabla 5. Valores de porosidad total de las muestras analizadas, obtenidas por intrusión de mercurio.

\begin{tabular}{|l|c|c|c|}
\hline & $O P C$ & $H s T$ 7\% & $H c G 7 \%$ \\
\hline Porosidad total (\%) & 18.46 & 18.86 & 18.83 \\
\hline
\end{tabular}

\subsection{Propiedades mecánicas de las pastas de cemento}

En las Figuras 6 y 7 están representados los resultados obtenidos para los ensayos de resistencia a compresión y a flexión a 2, 28 y 90 días de curador. En general, se observa que los cementos binarios presentan un comportamiento similar a todas las edades de hidratación, con una ligera disminución con respecto a la pasta OPC de referencia. A 2 días de curado, los valores mecánicos a compresión y flexotracción son más próximos al apasta de referencia, motivado por la presencia de mayor cantidad de calcita, la cual acelera la hidratación de las partículas de cemento para dar mono y/o hemi-carboaluminatos cálcicos rápidamente, aparte del efecto filler en ambos cementos [31]. 


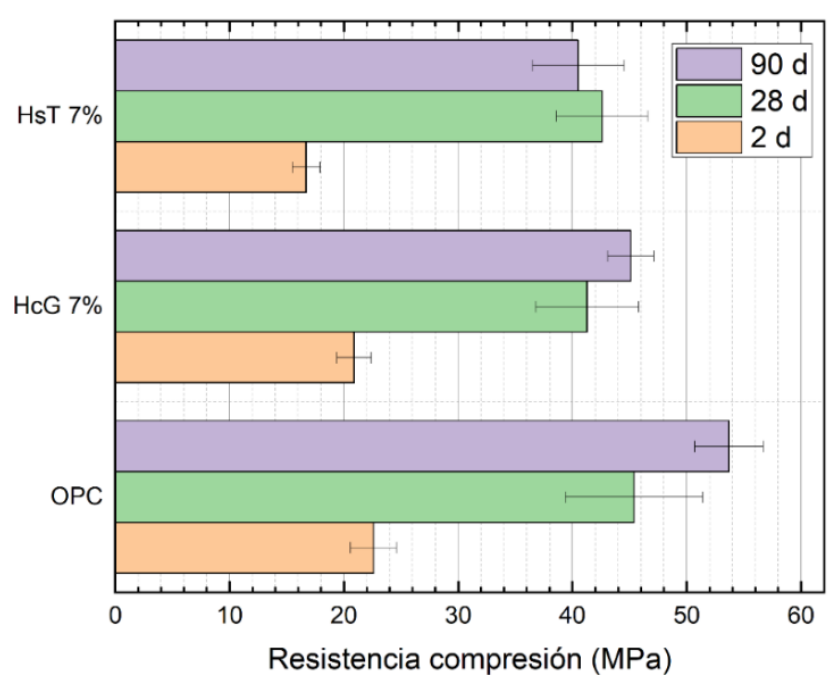

Figura 6. Resistencia a la compresión de todas las pastas de cemento a 2, 28 y 90 días de curado.

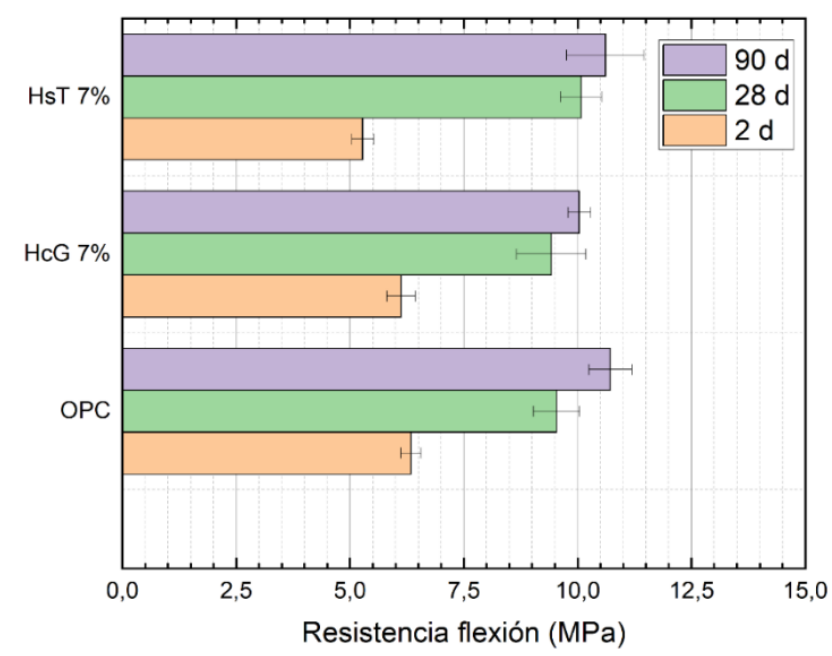

Figura 7. Resistencia a la flexión de todas las pastas de cemento a 2, 28 y 90 días de curado.

A partir de 28 días de curado, la ganancia de resistencia es menor que la experimentada por la pasta OPC mínima, lo que corrobora la baja actividad puzolánica de este tipo de residuos industriales (Fig. 3), por lo que predomina el efecto dilución del cemento OPC sobre la reacción puzolánica. Esta evolución encontrada en las resistencias mecánicas está en consonancia con los valores de microporosidad comentados anteriormente y están en línea con otras puzolanas de baja o media actividad puzolánica, como las escorias $\operatorname{SiMn[32]~y~residuos~de~granito[33].~}$

\section{CONCLUSIONES}

Las conclusiones más relevantes de la presente son las siguientes:

- Los residuos finos del hormigón reciclado (HsT y HcG) procedentes de RCDs, evidencian grandes diferencias químicas y mineralógicas en función de la naturaleza del árido empleado previamente en el elemento constructivo (calcáreo o silíceo).

- El residuo HsT, por su naturaleza ácida presenta una mayor reacción puzolanica que la mostrada por el residuo de naturaleza caliza. Atendiendo a los valores de la constante de 
reacción $(\mathrm{K})$, el residuo HsT muestra una reactividad 6 veces mayor que el de naturaleza calcárea.

- Las propiedades físicas de las pastas de cemento adicionadas con este tipo de residuos con un 7\% de sustitución son similares al del OPC, cumpliendo con los requerimientos normalizados, en cuanto al inicio de fraguado y expansión de volumen.

- La microporosidad de las pastas binarias es similar de referencia con poros mayoritariamente inferiores a 100 nanómetros, presentando en todos los casos el mismo porcentaje de porosidad total.

- Las propiedades mecánicas a compresión y flexotracción de las pastas de cemento binarias son ligeramente inferiores a la de la pasta de referencia, motivado más por el efecto dilución que por el de reactividad puzolánica. A pesar de ello, las diferencias son mínimas cumpliendo con los requerimientos mecánicos normalizados y manteniendo en todos los caos la misma categoría resistente que el cemento de partida, teniendo en cuenta las desviaciones obtenidas para probetas de $1 \times 1 \times 6 \mathrm{~cm}$.

A la vista de la presente investigación se puede resaltar que las fracciones finas del hormigón reciclado acumuladas en las plantas de gestión pueden ser viables como adiciones mineralógicas para la fabricación de futuros cementos eco-eficientes y sostenibles. Estos resultados obtenidos forman parte de un proyecto nacional vigente, cuyo objetivo final es la búsqueda de nuevas aplicaciones industriales de estos residuos, dirigidas fundamentalmente a la impresión 3D como futura tecnología de construcción.

\section{AGRADECIMIENTOS}

Este estudio ha sido financiado por el Ministerio de Ciencia, Innovación y Universidades (MICIU), por la Agencia Nacional de Investigación Española (AEI) y por la European Regional Development Fund (ERDF), bajo el proyecto RTI2018-097074-B-C21/22. Gracias también a la Asociación de RCDs Española (RCDA), Sika (Madrid, España) y al Instituto Español de Cementos y sus Aplicaciones (IECA).

\section{REFERENCIAS}

[1] I.F. Häfliger, V. John, A. Passer, S. Lasvaux, E. Hoxha, M.R.M. Saade, G. Habert, Buildings environmental impacts' sensitivity related to LCA modelling choices of construction materials, J. Clean. Prod. 156 (2017) 805-816.

https://doi.org/10.1016/j.jclepro.2017.04.052.

[2] T. Chinda, Investigation of factors affecting a construction waste recycling decision, Civ. Eng. Environ. Syst. 33 (2016) 214-226. https://doi.org/10.1080/10286608.2016.1161030.

[3] European Enviroment Agency, Construction and Demolition Waste : challenges and opportunities in a circular economy, Brief. No. 14/2019. (2020) 8. https://www.eea.europa.eu/publications/construction-and-demolition-wastechallenges/at_download/file.

[4] E.C.D.-G. Environment, EU Construction \& Demolition Waste Management Protocol, Off. J. Eur. Union. (2016) 1-22.

[5] European Commission, Directive 2008/98/EC of the European Parliament and of the Council on waste., (2008).

[6] F. Colangelo, T.G. Navarro, I. Farina, A. Petrillo, Comparative LCA of concrete with recycled aggregates: a circular economy mindset in Europe, Int. J. Life Cycle Assess. 25 (2020) 1790-1804. https://doi.org/10.1007/s11367-020-01798-6. 
[7] E. Moreno-Pérez, J. Hernández-Ávila, Y. Rangel-Martínez, E. Cerecedo-Sáenz, A. Arenas-Flores, M.I. Reyes-Valderrama, E. Salinas-Rodríguez, Chemical and mineralogical characterization of recycled aggregates from construction and demolition waste from Mexico city, Minerals. 8 (2018). https://doi.org/10.3390/min8060237.

[8] E. Asensio, C. Medina, M. Frías, M.I. Sánchez de Rojas, Fired clay-based construction and demolition waste as pozzolanic addition in cements. Design of new eco-efficient cements, J. Clean. Prod. 265 (2020). https://doi.org/10.1016/j.jclepro.2020.121610.

[9] H. Krour, R. Trauchessec, A. Lecomte, C. Diliberto, L. Barnes-Davin, B. Bolze, A. Delhay, Incorporation rate of recycled aggregates in cement raw meals, Constr. Build. Mater. 248 (2020). https://doi.org/10.1016/j.conbuildmat.2020.118217.

[10] M. Contreras, S.R. Teixeira, M.C. Lucas, L.C.N. Lima, D.S.L. Cardoso, G.A.C. da Silva, G.C. Gregório, A.E. de Souza, A. dos Santos, Recycling of construction and demolition waste for producing new construction material (Brazil case-study), Constr. Build. Mater. 123 (2016) 594-600. https://doi.org/10.1016/j.conbuildmat.2016.07.044.

[11] C. Medina, W. Zhu, T. Howind, M.I. Sánchez De Rojas, M. Frías, Influence of mixed recycled aggregate on the physical-mechanical properties of recycled concrete, J. Clean. Prod. 68 (2014) 216-225. https://doi.org/10.1016/j.jclepro.2014.01.002.

[12] P. Plaza, I.F. Sáez del Bosque, M. Frías, M.I. Sánchez de Rojas, C. Medina, Use of recycled coarse and fine aggregates in structural eco-concretes. Physical and mechanical properties and CO2 emissions, Constr. Build. Mater. 285 (2021) 122926. https://doi.org/10.1016/j.conbuildmat.2021.122926.

[13] Instrucción Española de Hormigon Estructural, 2021.

[14] I. Vegas, I. Azkarate, A. Juarrero, M. Frías, Design and performance of masonry mortars made with recycled concrete aggregates, Mater. Constr. 59 (2009) 5-18. https://doi.org/10.3989/mc.2009.44207.

[15] J. Moreno-Juez, I.J. Vegas, M. Frías Rojas, R. Vigil de la Villa, E. Guede-vázquez, Laboratory-scale study and semi-industrial validation of viability of inorganic CDW fine fractions as SCMs in blended cements, Constr. Build. Mater. 271 (2021) 121823. https://doi.org/10.1016/j.conbuildmat.2020.121823.

[16] M. Frías, R.V. de la Villa, S. Martínez-Ramírez, L. Fernández-Carrasco, E. Villar-Cociña, R. García-Giménez, Multi-Technique Characterization of a Fine Fraction of CDW and Assessment of Reactivity in a CDW/Lime System, Minerals. 10 (2020) 590. https://doi.org/10.3390/min10070590.

[17] European Commission, Circular economy action plan, Eur. Comm. (2020) 28. https://doi.org/10.2775/855540.

[18] M. Frias, R. Garcia-Gimenez, S. Martinez-Ramirez, J. Moreno-juez, M. Monasterio, L. Caneda-Martínez, Behaviour and Properties of Eco-Cement Pastes Elaborated with Recycled Concrete Powder from Construction and Demolition Wastes, Materials (Basel). 14 (2021) 1299. https://doi.org/https:// doi.org/10.3390/ma14051299.

[19] EN 196-1:2016 - Methods of testing cement - Part 1: Determination of strength, n.d. https://standards.iteh.ai/catalog/standards/cen/37b8816e-4085-4dcc-a642a383d9bddd6c/en-196-1-2016 (accessed August 3, 2021).

[20] A. Koch, U. Steinegger, A rapid test for cements for their behaviour under sulphate attack., Zem-Kalk-Gips. 7 (1960) 317-324.

[21] M. Frías, R. García, R. Vigil, S. Ferreiro, Calcination of art paper sludge waste for the use as a supplementary cementing material, Appl. Clay Sci. 42 (2008) 189-193. https://doi.org/10.1016/j.clay.2008.01.013.

[22] CEN-CENELEC, European Standard EN 196-3:2016. Methods of testing cement-Part 3: determination of setting times and soundness, Brussels, Belgium, 2016. 
[23] UNE-EN 196-1 Parte 1 : Determinación de resistencias, 2018.

[24] M. Frías, M.I. Sánchez de Rojas, M.P. Luxán, N. García, Determination of specific surface area by the laser diffraction technique. Comparison with the blaine permeability method, Cem. Concr. Res. 21 (1991) 709-717. https://doi.org/10.1016/0008-8846(91)90165-E.

[25] C. Ulsen, H. Kahn, G. Hawlitschek, E.A. Masini, S.C. Angulo, V.M. John, Production of recycled sand from construction and demolition waste, Constr. Build. Mater. 40 (2013) 1168-1173. https://doi.org/10.1016/j.conbuildmat.2012.02.004.

[26] M. Frías, O. Rodríguez, I. Vegas, R. Vigil, Properties of calcined clay waste and its influence on blended cement behavior, J. Am. Ceram. Soc. 91 (2008) 1226-1230. https://doi.org/10.1111/j.1551-2916.2008.02289.x.

[27] E. Villar-Cociña, E.V. Morales, S.F. Santos, H. Savastano, M. Frías, Pozzolanic behavior of bamboo leaf ash: Characterization and determination of the kinetic parameters, Cem. Concr. Compos. 33 (2011) 68-73. https://doi.org/10.1016/j.cemconcomp.2010.09.003.

[28] M. Frías-Rojas, M.I. Sánchez-de-Rojas-Gómez, C. Medina-Martínez, E. Villar-Cociña, New trends for nonconventional cement-based materials: Industrial and agricultural waste, 2017. https://doi.org/10.1016/B978-0-08-102001-2.00007-3.

[29] CEN-CENELEC, European Standard EN 197-1:2011. Cement - Part 1: Composition, specifications and conformity criteria for common cements, Brussels, Belgium, 2011.

[30] T. Vuk, V. Tinta, R. Gabrovšek, V. Kaučič, The effects of limestone addition, clinker type and fineness on properties of Portland cement, Cem. Concr. Res. 31 (2001) 135-139. https://doi.org/10.1016/S0008-8846(00)00427-0.

[31] T. Oey, A. Kumar, J.W. Bullard, N. Neithalath, G. Sant, $\uparrow \dagger$ Member, The Filler Effect: The Influence of Filler Content and Surface Area on Cementitious Reaction Rates, n.d. https:/www.nist.gov/publications/filler-effect-influence-filler-content-and-surface-areacementitious-reaction-rates (accessed February 12, 2021).

[32] M. Frías, C. Rodríguez, Effect of incorporating ferroalloy industry wastes as complementary cementing materials on the properties of blended cement matrices, Cem. Concr. Compos. 30 (2008) 212-219. https://doi.org/10.1016/j.cemconcomp.2007.05.004.

[33] G. Medina, I. Saez del Bosque, M. Frías, M.I. Sanchez de Rojas, C. Medina, Effect of granite waste on binary cement hydration and paste performance: statistical analysis, Mater. J. 116 (2019) 63-72. 